\title{
SHEAVES IN THE BRAIN ELUCIDATE THE BEHAVIOR OF ENTRAINED OSCILLATIONS
}

\author{
Arturo Tozzi \\ Center for Nonlinear Science, Department of Physics, University of North Texas, Denton, Texas 76203, USA \\ 1155 Union Circle, \#311427, Denton, TX 76203-5017 USA \\ tozziarturo@libero.it \\ Arturo.Tozzi@unt.edu
}

Once a wheat sheaf has been sealed and tied up, its packed down straws display the same orientation and zerodivergence. This observation brings us to the mathematical notion of presheaf, i.e., a topological structure in which diverging functions are locally superimposed. We show how the concepts of presheaves and the correlated globular sets, borrowed from category theory and algebraic topology, allow a well-founded mathematical approach to otherwise elusive activities of the brain. The mathematical assessment of brain functions in terms of presheaves: a) explains why spontaneous random spikes synchronize; b) leads to the counterintuitive intuition of antidromic effects in neuronal spikes: when an entrained oscillation propagates from A to B, changes in B lead to changes in A. We provide testable previsions: a) we suggest the proper locations of transcranial magnetic stimulation's coils to improve the clinical outcomes of drug-resistant epilepsy; b) we advocate that axonal stimulation by external sources backpropagates and alters the neuronal electric oscillatory frequency. Further, we describe how the hierarchical information transmission inside globular sets provides fresh insights concerning different issues at various coarse-grained scales, such as object persistence, memory reinforcement in spite of random noise, Bayesian inferential circuits.

KEYWORDS: globular set; category theory; multidimensional; visual recognition; drug-resistant epilepsy; transcranial magnetic stimulation.

The brain activity has been recently tackled through mathematical/topological weapons, such as algebraic topology, near set theory and category theory (Tozzi et al., 2017a; Peters et al., 2017). To make an example, Northoff et al. (2019) investigated the relationships (the authors call them "the sameness") between consciousness and its neural correlates in terms of category theory, composition of functions and functors. However, these theoretical approaches to the brain are constrained by soaring abstraction and vagueness in defining regular structures and nearness relations (Wolski 2013), thus preventing the formulation of sharp experimental previsions. The main goal of this paper is to overcome these difficulties, providing a mathematical framework that allows a quantifiable description and assessment of neuroscientific issues. We focus on one of the simplest category theoretic structures, i.e., the so-called pre-sheaf (Bredon 1997), that is rich enough to convey a feasible explanation of brain function. To provide an example of presheaves for non technical readers, think to a sheaf of wheat, sealed and tied up with twine supply. The countless diverging straws with various orientation are compacted in well-ordered tiny sections, where they display coherent behavior and matching description (Figure 1A). To provide another example, it is noteworthy that a neuroscientific technique, i.e., the tractography, allows the identification of well-defined anatomical white matter structures that resemble presheaves (Figure 1B).

Presheaves offer invaluable benefits: a) they generalize the local systems that are so ubiquitous in mathematics; b) have powerful applications to the topology of algebraic and the analytic complex varieties of singular spaces (Dimca, 2004); c) make available a suitable notion of "general coefficient systems"; d) stand for common methods of comparison between different cohomology theories of general topological spaces (Bredon, 1997); provide foundations of near sets (Wolski 2013). Here we show how presheaves give us the possibility to treat neuroscientific issues in terms of category theory, in particular using composition of functions/globular sets, and to draw previsions that can be experimentally tested with the current neuro-technologies. In particular, globular sets lead to a counterintuitive claim: when an entrained oscillation propagates from the brain area A to a brain area B, changes in spike frequency in B lead to changes in spike frequency in A. Also, globular sets allow to analyze the stability of synchronous states in dynamical systems such as the brain (Papo and Buldú, 2019), shedding new light of the still unknown mechanisms of spike synchronization. 
A
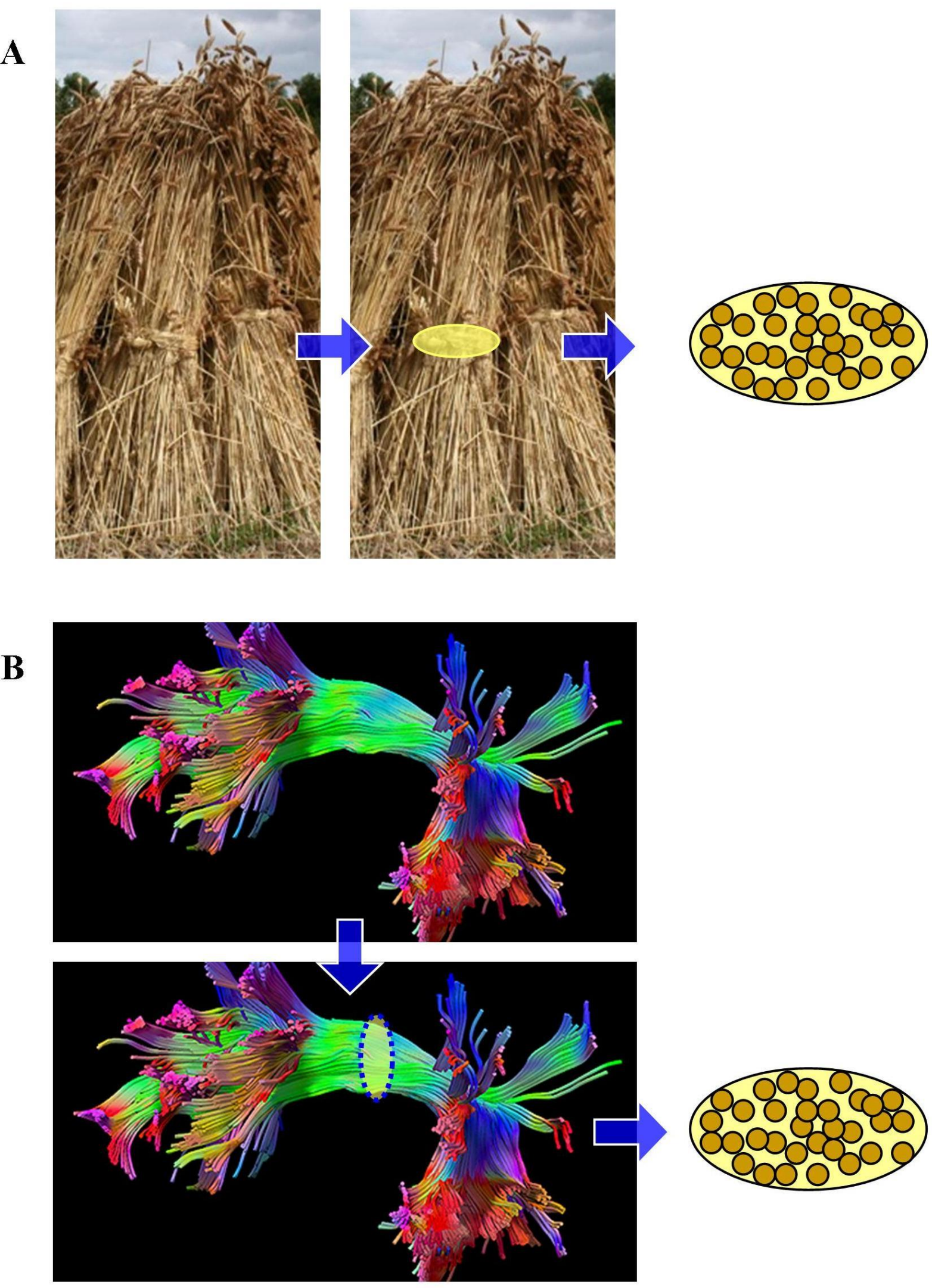

Figure 1. Examples of presheaves-like structures in the real world. Figure 1A: a wheat sheaf displays a cross-section in which the straws, rather being scattered, are very close one each other. Modified from: https://sadeik.wordpress.com/blog/about/corn-dolly-making/. Figure 1B: diffusion MRI maps white matter bundles and assesses their structural integrity. Note that the white matter bundles display close analogy with the wheat straws depicted in Figure 1A. Modified from: http://brain.labsolver.org/. 


\section{GLOBULAR SETS: A TECHNICAL APPROACH}

At first, we will describe the mathematical apparatus that will be used in the sequel to describe nervous functions. In particular, we will talk about composition of functions, globular sets, presheaves.

Composition of functions. When working with a specific input value (named either $\mathrm{x} \in \mathrm{X}$, or another letter), the composition of functions (denoted by the symbol o):

is defined by:

$$
\mathrm{g} \circ \mathrm{f}(\mathrm{x})
$$

$$
(g \circ f)(x)=g(f(x)) .
$$

In other words, take the function $f(x)$ and put it in place of $x$ in the function $g$, so that the output of $f$ is used as the input of $g$ (Bergman 2011). In case of two functions:

then gof is a function from $\mathrm{X}$ to $\mathrm{Z}$, so that:

$$
f: X \rightarrow Y \text { and } g: Y \rightarrow Z,
$$$$
\text { gof: } X \rightarrow Z \text {. }
$$

Globular sets: presheaves come into play. A globular set is a geometric shape for higher structures, standing to simplicial sets as globes to simplices. The category of globes is a category whose objects are natural numbers, and whose morphisms are generated from:

$$
\begin{aligned}
& \sigma \mathrm{n}:[\mathrm{n}] \rightarrow[\mathrm{n}+1] \\
& \tau \mathrm{n}:[\mathrm{n}] \rightarrow[\mathrm{n}+1]
\end{aligned}
$$

A globular set is a presheaf on the category of globes (Kashiwara and Schapira, 1994; Bredon 1997), i.e., a particular kind of contravariant functor which helps to capture sections:

$$
\text { gSet:=PSh(G) }
$$

To achieve a presheaf $F$ in the topological space W (standing for our universe of discourse), two requirements are needed: gluability and uniqueness (Street 2000; Simpson 2011).

Once these requirements are satisfied, for each open $\mathrm{X} \subset \mathrm{W}$, the restriction map is a homeomorphism

such that $\rho_{\mathrm{w}, \mathrm{w}}$ is an identity.

$$
\rho_{\mathrm{w}, \mathrm{X}}: F(\mathrm{~W}) \rightarrow F(\mathrm{X}),
$$

Therefore, in terms of the category of globes, the globular set $X \in g$ Set is a sequence of sets $X_{0}, X_{1}, X_{2}, . ., X_{n}$, i.e., a collection of the set of $n$-globes $\left\{X_{n}\right\}_{n \in N} \subset W$, that is equipped with pairs of functions:

such that the following globular identities hold:

$$
\left\{s_{n}, t_{n}: X_{n+1} \rightarrow X_{n}\right\}_{n \in N}
$$

$$
\begin{gathered}
\mathrm{s}_{\mathrm{n}} \circ \mathrm{S}_{\mathrm{n}+1}=\mathrm{S}_{\mathrm{n}} \circ \mathrm{t}_{\mathrm{n}+1} \\
\quad \text { and } \\
\mathrm{t}_{\mathrm{n}} \circ \mathrm{S}_{\mathrm{n}+1}=\mathrm{t}_{\mathrm{n}} \circ \mathrm{t}_{\mathrm{n}+1},
\end{gathered}
$$

where $\mathrm{s}$ and $\mathrm{t}$ stand for the two sections termed, respectively, source and target (Figure 2A).

Note that $X_{n}$ may also stand for directed edges at level $n$.

Further, another constraint is required: no reflexive globular sets do exist, or, in other words, do not exist degeneracy maps going back in the opposite direction $X_{1} \rightarrow X_{2}$ (Simpson 2011).

In the next paragraphs, presheaves will be used to skecth a theory that describes in general terms the nervous functions taking place in cortical tissues.

\section{BUILDING NERVOUS PRESHEAVES}

In this paragraph we will analyze nervous activity from the standpoint of presheaf theory. Take a brain area W, equipped with two functions termed $\mathrm{s}$ and $\mathrm{t}$ (Figure 2). They diverge everywhere, apart from a cortical subarea (i.e., a subset of $W$ ) encompassing $X_{1}$ and $X_{2}$, with $X_{1} \cap X_{2}$. If in the subarea $X_{1} \cap X_{2}$, the above-mentioned constraints are satisfied so that the topological concept of presheaf holds, the functions $s$ and $t$ display an unusual behavior. The two functions $s$ and $t$ do not diverge inside the subsets $X_{1}$ and $X_{2}$, rather they display coherence, equality and matching description. Indeed, globular sets and presheaf theory require that the composition of functions occurring inside the subset $X_{1} \cap X_{2}$ does not diverge as it does in other subsets. Furthermore, the functions $s_{2}$ and $t_{2}$ in $X_{2}$ precede the functions $s_{1}$ and $t_{1}$ in $X_{1}$ : this means that the output of the cortical subarea $X_{2}$ is used as the input of the cortical subarea $X_{1}$. Therefore, changes in functions occurring in $X_{2}$ lead to corresponding changes in functions occurring in $X_{1}$. In 
general terms, we can state that source and targets in $\mathrm{X}_{1}$ follow sources and targets in $\mathrm{X}_{2}$. Also, there is an equivalence between:

1) $X_{1}$ sources that follow $X_{2}$ sources and $X_{1}$ sources that follow $X_{2}$ targets;

2) $X_{1}$ targets that follow $X_{2}$ sources and $X_{1}$ targets that follow $X_{2}$ targets;

3) $X_{1}$ targets that follow $X_{2}$ sources and $X_{1}$ targets that follow $X_{2}$ targets.

Is brief, $\mathrm{X}_{2}$ functions (source and target) point towards and modify $\mathrm{X}_{1}$ functions (source and target). Therefore, $\mathrm{X}_{2}$ controls $\mathrm{X}_{1}$, and not vice versa.

Summarizing, the brain activity assessed in terms of presheaves/globular sets explains two features of neural functions (Figures 2B-2C):

1) it elucidates why brain oscillations equipped with different spike frequency and amplitude tend to converge and coalesce towards single functional areas, where overlapping and matching synchronized frequencies/amplitudes contribute to keep the signal steady (Figure 2B).

2) Second, it suggests that a hierarchical oscillatory activation occurs inside single functional areas, where the oscillations are constrained to follow strict temporal sequences: this means that, when an entrained oscillation propagates from A to B, a change in B frequency leads to a change in A frequency (Figure 2C).

This last, counterintuitive observation has foremost implications in the study of the brain activity, as we will shall see in the next paragraphs. 
A
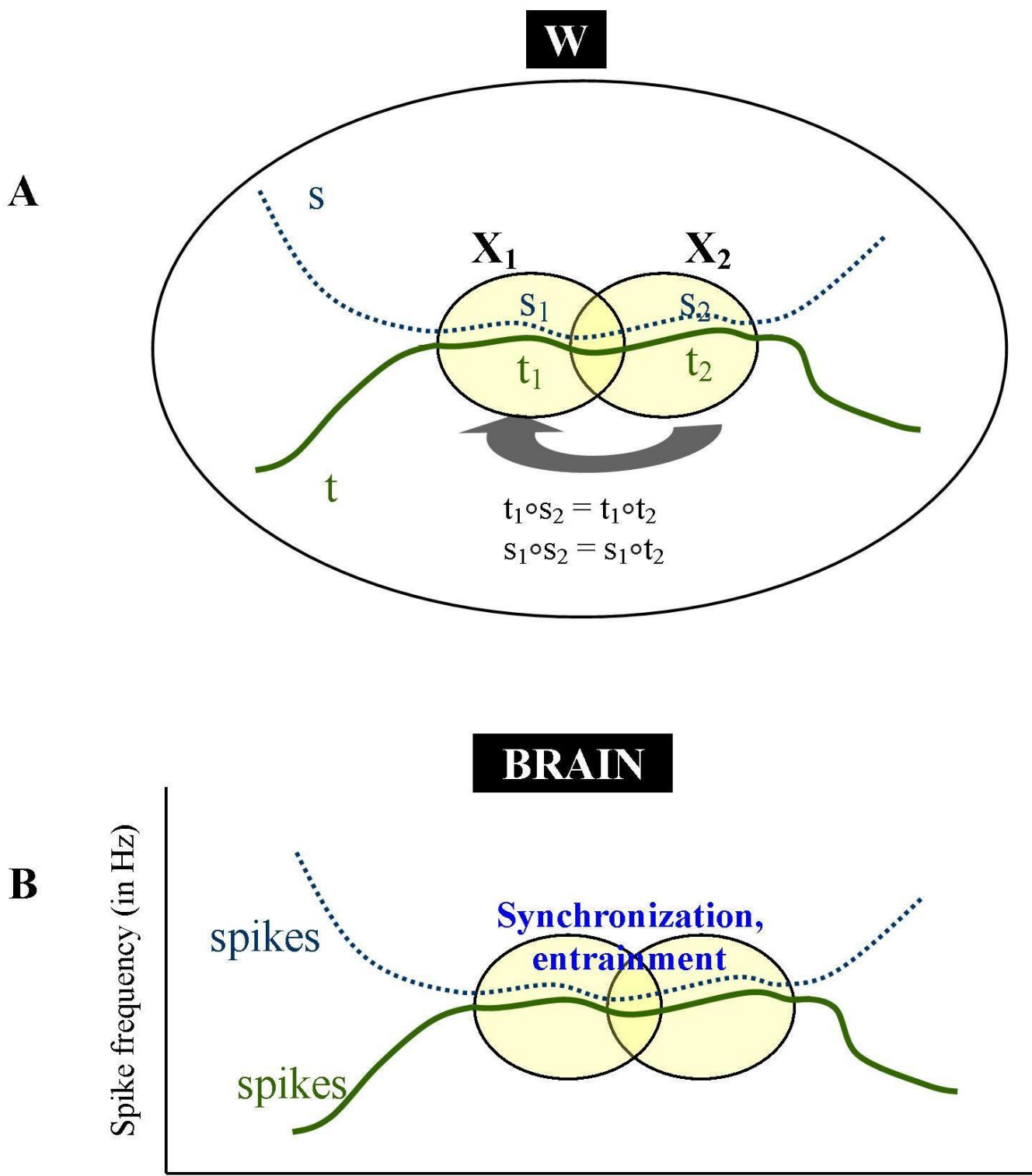

Different brain locations

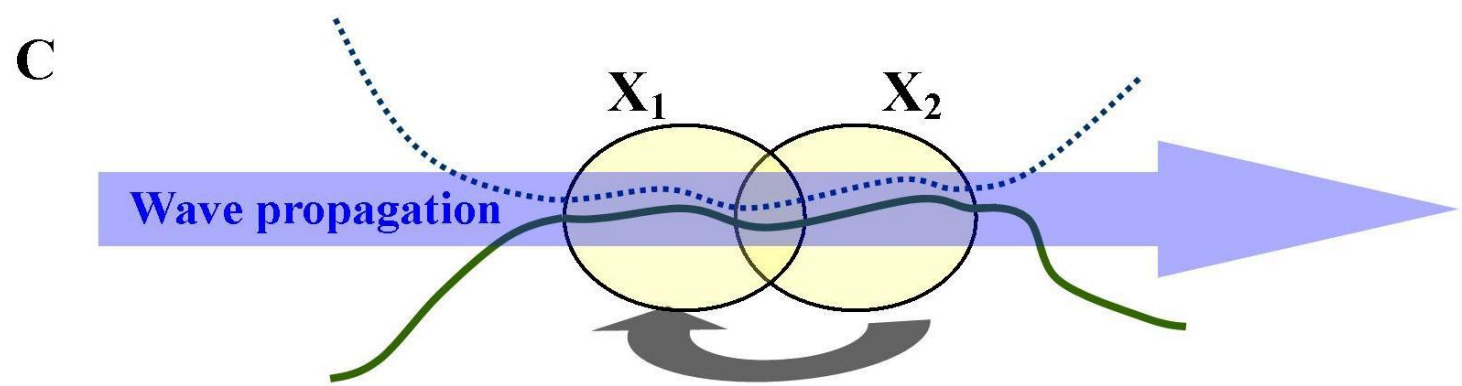

$\mathrm{X}_{2}$ modifies $\mathrm{X}_{1}$

Figure

2A. Geometric counterparts of the rather abstract concept of globular sets/presheaves, in the universe of discourse W of category theory. The same nomenclature of the main text is used. For further details, see: https://ncatlab.org/nlab/show/globular+set\#references Figures 2B: concerning the universe of discourse termed "brain", globular sets allow to explains how and why spikes of different incoherent frequencies are synchronized in certain brain areas. Furthermore, a nervous globular sets' theory suggests that antidromic spikes do exist (arrow), so that $\mathrm{X}_{2}$ exerts effects on $\mathrm{X}_{1}$ (Figure 2C). 


\section{PRESHEAVES AND GLOBULAR SETS: TESTABLE PREVISIONS IN NEUROSCIENCE}

Once described the mathematical apparatus of presheaves and globular sets, we will provide here theoretical examples of feasible applications in neuroscience, suggesting previsions that are testable with the current technology.

Coil location for transcranial magnetic stimulation in epilepsy. Transcranial magnetic stimulation (TMS) is a focal electrical brain stimulation induced by powerful magnetic fields (Chung et al., 2016; Jan et al, 2017; Kohli and Casson, 2019). Protocols may be divided into single-pulse, paired-pulse, and repetitive TMS (rTMS). While specific high frequency rTMS has been approved for the treatment of major depression, low frequency rTMS $(0.3-1 \mathrm{~Hz})$ is able to decrease cortical excitability, suggesting a potential therapeutic effect for patients with drug-resistant epilepsy (Jan et al., 2017). Despite conventional rTMS stimulators activate only superficial cortical areas, it is possible to reach deep epileptic foci (such as, for example, in temporal lobe epilepsy), using specially designed H-coils that provide low frequency rTMS (Gersner et al., 2016). Chen et al. (2016) retrospectively analyzed the evidence for the efficacy of TMS in drug-resistant epilepsy, compared with available treatments. They evaluated studies that used rTMS of any frequency, duration, intensity and setup (focal or vertex treatment) on patients of different ages. Given the extreme variability in technique and outcome reporting, the evidence for efficacy of rTMS for reduction in seizure rate from baseline rate, in seizure frequency and in mean number of epileptic discharges, is still lacking (Jan et al., 2017). The main problem is that there is still no agreement on optimized stimulation parameters and patterns of rTMS for epilepsy. Indeed, TMS effects are erratic across individuals and depend on frequency, number of stimuli within a train, stimulation intensity, type of coil, coil position, duration of stimulation, and inter-train interval.

In case of epileptic seizures, our model hypothesizes that the area of onset of pathological spikes might extend to other regions through a mechanism of synchronization linked with the transient formation of globular sets. If we consider synchronous pathological spikes as taking place inside functional globular sets, we achieve a novel mathematical framework for epilepsy that is worth to be investigated. Indeed, if the presheaf framework holds true, the starting epileptic focus (standing for $\mathrm{X}_{1}$ ) propagates to the surrounding brain areas (standing for $\mathrm{X}_{2}$ ), giving rise to synchronized pathological waves with matching description/zero divergence (Figures 3A-B). The areas $\mathrm{X}_{2}$ are therefore able to modify the oscillatory activity of the starting locus $X_{1}$ of epilepsy onset. This means that the areas surrounding the locus of seizure onset keep the seizure active, and not vice versa. This provides us with an easily testable prevision: to achieve the best therapeutic effect and confirm that the mathematical apparatus of globular sets holds true in epilepsy, rTMS treatments must not focus on the first focus of onset of epilepsy, rather on the surrounding areas where the pathological spike propagates (Figure 3B).

Feedback and feedforward projections. It is well-known that feedforward pathways are associated with enhanced gamma $(30$ to $70 \mathrm{~Hz})$ oscillations, whereas feedback projections, that account for a large portion of all connections between neurons in the thalamocortical system, selectively modulate alpha/low-beta ( 8 to $15 \mathrm{~Hz}$ ) oscillations (Mejias et al., 2016; Michalareas et al., 2016; Sikkens et al., 2019). The interplay between hierarchically organized feedforward (bottom-up) and feedback (top-down) signaling involves intricate dynamics across multiple (intralaminar, interlaminar, interareal, and whole cortex) scales involved in attentional processes, predictive coding, executive control (Mejias et al., 2016). Information flows through visual areas in opposite directions during bottom-up intake of current stimuli and top-down processes of memory or attention (Bastos et al., 2015; Zheng and Colgin, 2015; Michalareas et al., 2016). Also, in working memory and its volitional control, an interplay occurs between network gamma oscillations in superficial cortical layers, and alpha and beta in deep cortical layers (Miller et al., 2018). To provide an example, Figure 3C describes the case of prediction and errors signals in the Bayesian brain. In terms of presheaf theory, this means that a change in frequency occurring in the cortical location $\mathrm{X}_{2}$ leads also to a change in frequency in the cortical location $\mathrm{X}_{1}$. This means that the electric frequency of the brain is also controlled antidromically: along an oscillatory pathway, the distal frequency is able to modify the proximal frequency. 
A
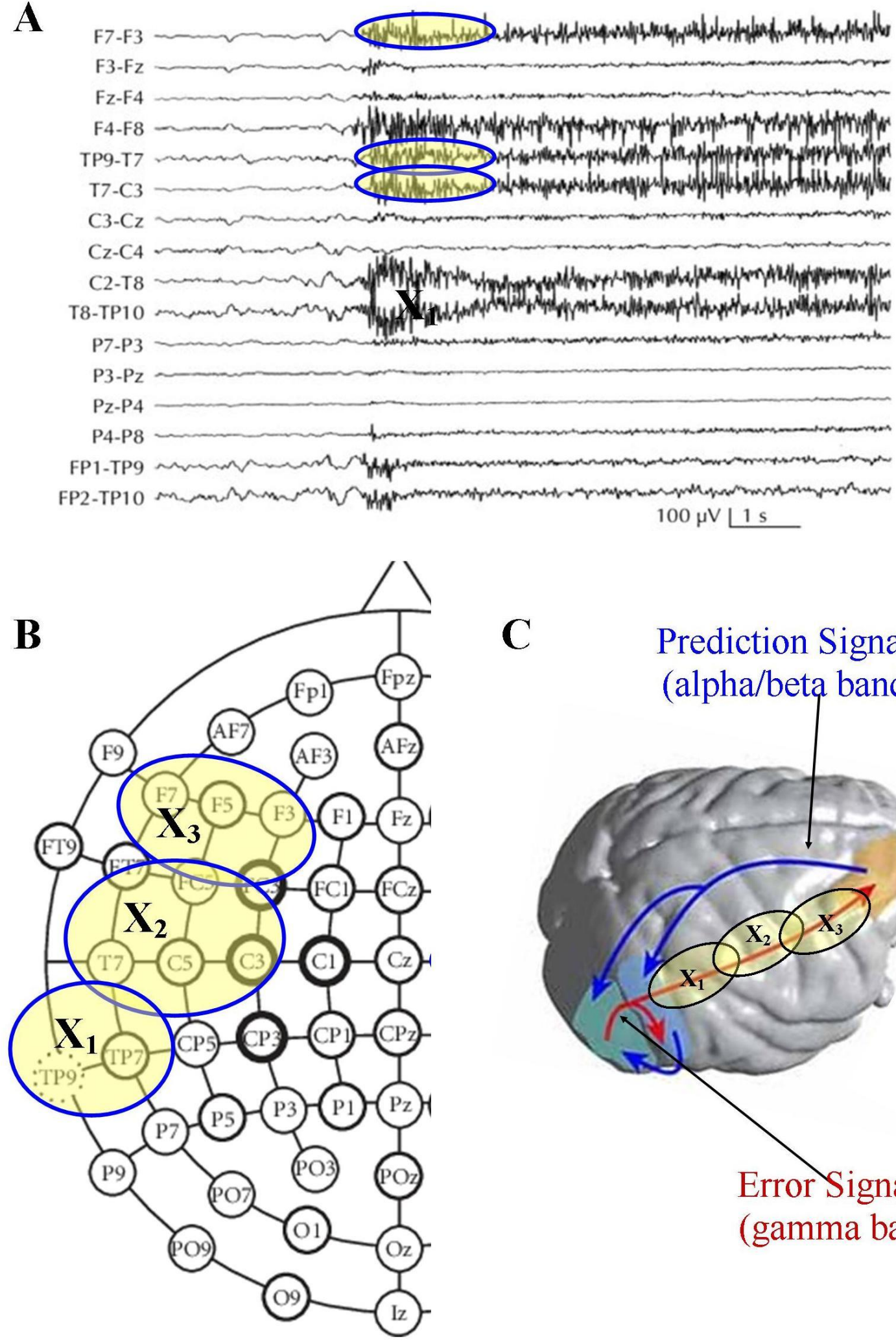

C

Prediction Signals (alpha/beta band)

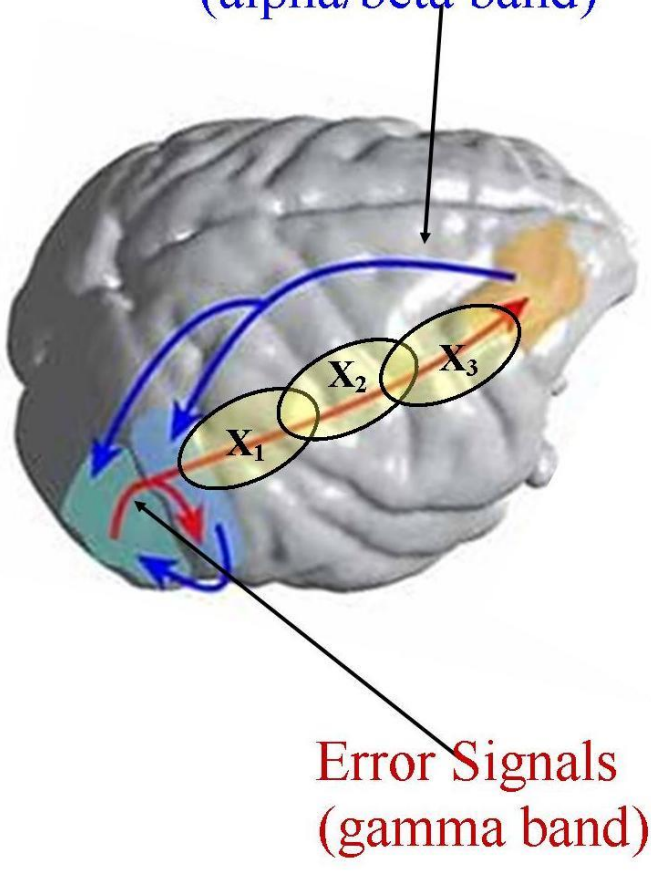

Figure 3A. Recorded surface EEG (displayed in a bipolar transverse montage) showing a left parietal EEG seizure onset. Modified from: Beleza et al. (2010). Figure 3B. The EEG mount illustrates the globular sets involved in this seizure. Modified from: Tangermann et al. (2005). Figure 3C. An example of feedforward and feedback projections in the brain. The globular sets corresponding to the gamma feedforward spikes are superimposed. Modified from: Sikkens et al. (2019). See text for further details. 
External brain stimulation. Presheaf model might help to describe the neurophysiology of brain stimulation by external sources. Extracellular electromagnetic stimulations produce electrical fields in both myelinated and unmyelinated axons (Rattay F. 1986; Basser and Roth, 1991), generating hyperpolarized regions of the cell membrane (Rattay et al., 2012). It is well-known that intracranial cortical stimulation induces selective activation across cell types and layers (Komarov et al., 2019), while external brain gamma stimulation modulates motor performance/GABA-Aergic interneuron activity (Guerra et al., 2018; McDermott et al., 2018). Nevertheless, origin, cellular mechanisms, control and functional effects of rTMS are unidentified (Benali et al., 2011). At present, there is no systematic method to predict which neural elements will be activated by a given stimulation regime (Komarov et al., 2019), because each individual has different rhythmic firing pattern in response to burst stimulation (Chung et al., 2019) and spike initiation site may change for short and long pulses (Rattay et al., 2012).

Our model sheds new light on the mechanisms of rTMS, suggesting that axonal stimulations from external sources might modify the frequency charges of the involved neurons. Figure 4 provides a theoretical example of a neuron (or a neuronal assembly) that fires at, say, $30 \mathrm{~Hz}$. If we use a $10 \mathrm{~Hz}$ current applied across its own axon, our model predicts that this neuron will synchronize its output frequency towards $10 \mathrm{~Hz}$. In other worlds, we suggest that a direct axonal stimulation antidromically backpropagates towards the neuronal bodies, due to the mathematical constraints of presheaves. Despite our scarce knowledge of antidromic oscillations' effects on neural information processing, experimental data point towards the possibility that axon modulation affects frequency encoding in neurons, before and after it is convoyed. Axons are not only information carriers, rather their trunks are endowed with ionotropic and metabotropic receptors for transmitters/neuromodulators (DeMaegd and Stein, 2018). Neuromodulatory effects on axons generate ectopic spiking, characterized by ion potentials backpropagating towards different sites, independent of incoming sensory or synaptic events. In particular, increasing ectopic spike frequency leads to delayed peripheral bursts, reductions in spike number/burst duration, and modifications in sensory firing frequency. Also, computational models demonstrate that antidromic action potentials are capable of altering sensory encoding in neurons with slow ionic conductances (DeMaegd and Stein, 2018). During $\gamma$-tACS over the right temporal lobe, Santamecchi et al. (2019) observed an EEG increase in $\gamma$ spectral power over bilateral temporal lobes after stimulation, leading to functional connectivity of bilateral temporal lobes. Thus, axonal neuromodulation, in the context of a mathematical approach framed on globular set theory, might have the role to produce quick frequency changes also in sites away from the stimulated axons. 
A

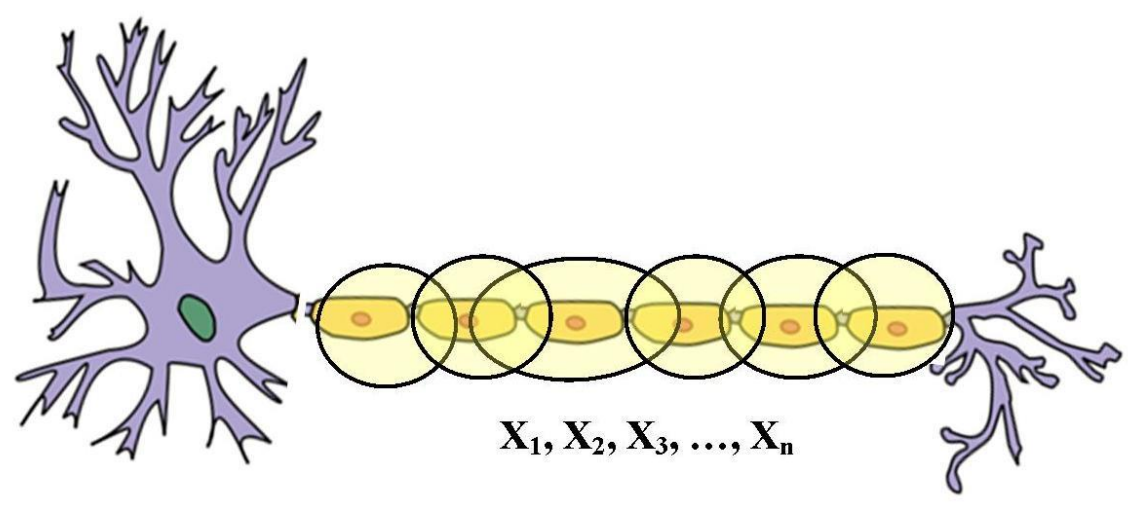

B

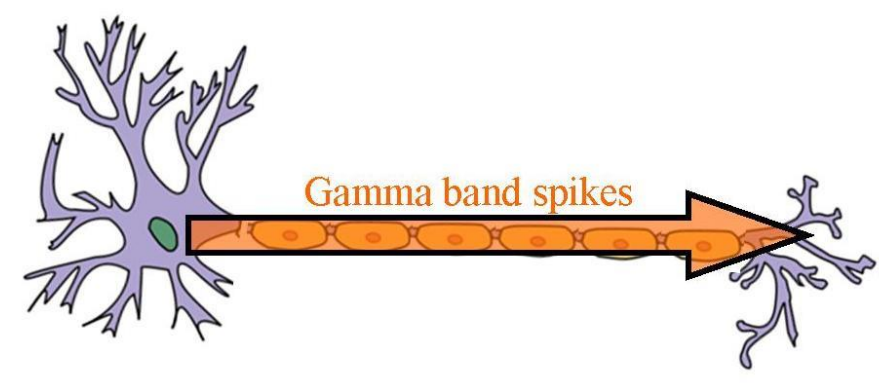

C

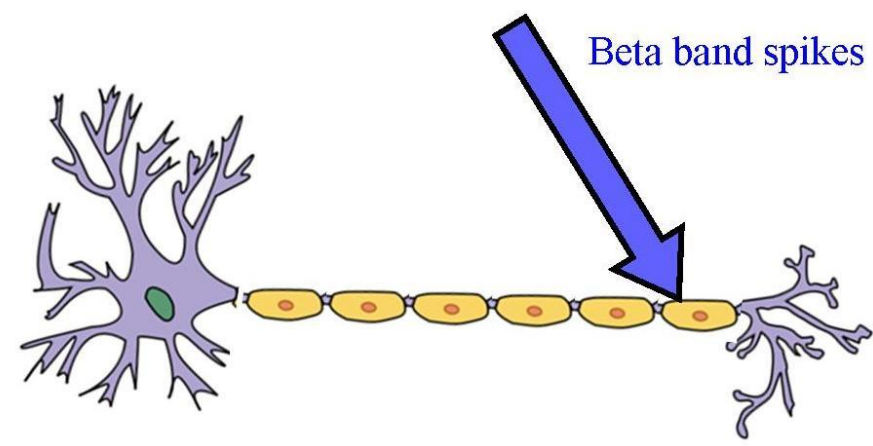

D

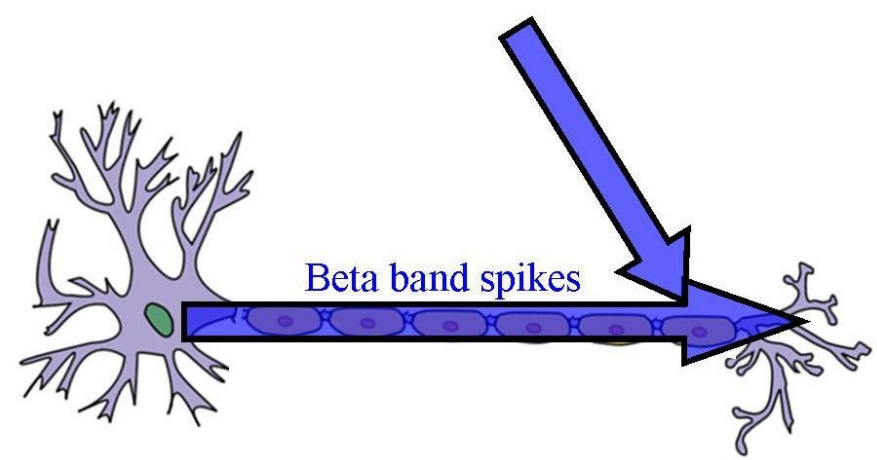

Figure 4. A testable prevision to check whether presheaves do exist in the brain. Figure 4A: according to presheaf models, the neuronal axon must be equipped with globular set's intersecting subsets. Figures 4B-D provide an example. Take a neuron that produces gamma bursts (Figure 4B). According to presheaf theories, if a continuous distal beta spike is applied to the axon (Figure 4C), the neuron will proximally generate entrained beta bursts, instead of the previous gamma bursts (Figure 4D). 


\section{CONCLUSIONS: MORE ABSTRACT POSSIBILITIES}

We described the mathematical apparatus of presheaves /globular sets and provided theoretical examples of feasible applications in neuroscience. Apart the ones described above, other more theoretical applications in neural systems can be hypothesized. The size of the globular sets may vary, so that presheaves can be used for the description of different coarse-grained nervous structures, both at the micro- and at the macro-levels of observation. Human ability to reidentify objects through time and across gaps in observation leads to the effect termed object persistence (Fields, 2013). The computational task of object persistence can be tackled in terms of presheaf theory, both at the microscopic level of histological microcolumns (Tozzi et al., 2017b) and at the macroscopic level of brain functional and anatomical areas. Concerning micro-levels of observation, globular sets could be located inside the multilayer arrangement of the cortex. In particular, presheaves might be endowed in the layer IV of the primary sensory occipital areas of the Primates, where large amounts of granular cells process the visual information coming from the thalamus (Figure 5A). In this milieu, the role of the globular sets' enduring coalescence of functions would be threefold: to synchronize oscillatory patterns, to stabilize information content and to produce unambiguous, long-lasting percepts. Concerning the macro-levels of brain analysis, it is well known that the face-selective response to static images of famous and unfamiliar faces is mediated by neural activity in the occipital/posterior temporal cortex, across the fusiform face area (FFA) (Axelrod et al., 2019). FFA recognizes faces, despite the underlying noise and the huge amount of conflicting inputs: the brain will be always able to recognize Jennifer Aniston, independent of her dresses, make-up or haircut. If we consider FFA in terms of a sheaf $F$ equipped with, say, globular sets $X_{1} \cap X_{2}$, a change occurring in $X_{2}$ will also involve $X_{1}$. Because the FFA subareas $X_{1}$ and $X_{2}$ are synchronized, they must always display matching description. This ensures stability to the percept and explains why face recognition is always constant, despite the changes in the environmental inputs. A presheaf approach might help to elucidate the issue of memory too. Indeed, memory could be stored in globular sets, that allow preservation and flexibility of the information stored inside them. Memories, (although scattered throughout the brain, if the theory of sparce code holds true), could be encompassed in intersecting globular sets, so that their content is represented more than once (in $X_{1}, X_{2}, \ldots, X_{n}$ ). Therefore, the otherwise labile stability of information content is granted by the globular set apparatus endowed in the universe of discourse $\mathrm{W}$, where the ubiquitous divergence among the functions equals zero.

The brain as an inferential machine. In Bayesian theories, that consider the brain an inferential machine, the required priors can be mathematically assessed in terms of presheaves, that have the role of keeping information stable. Indeed, two subsets $\mathrm{X}_{1}$ and $\mathrm{X}_{2}$ with $\mathrm{X}_{1} \cap \mathrm{X}_{2}$ could stand not just for two "spatially" separated structures, but also for two "temporally" separated events, i.e., two ensuing temporal windows. In this temporal case, due to the dictates of globular sets, it is required that the event $X_{1}$ at time $t_{1}$ follows the event $X_{2}$ at time $t_{2}$. This means that the events in $t_{2}$ are able to influence the events in $t_{1}$. This counterintuitive claim does not break any physical law, if we think to priors in terms of mental activities such as predictions, volitions, goals. When the event predicted by mental activities occurs in the external world, this means that $\mathrm{X}_{1}$ becomes real. Therefore, we are allowed to state that the real event follows from the Bayesian prior, because the previous prediction $\mathrm{X}_{2}$ causes the subsequent event $\mathrm{X}_{1}$ to occur. This mathematical approach ensues an answer to one of the most controversial philosophical issues concerning the mind/world relationships: presheaves explain how the elusive, immaterial mental activity is able to provoke physical effects in the real world.

The multidimensional brain. We stated that source and targets in $X_{n}$ follow sources and targets in $X_{n+1}$. This leads to another intriguing possibility. If we take the value " $n$ " in terms of "spatial" dimensions of the subtending manifold, we are allowed to investigate a recently-raised hypothesis, i.e., that the brain activity takes place in multidimensional spaces (Tozzi and Peters 2016, Tozzi 2019). Globular sets and presheaves provide us a potential method to localize the elusive higher-dimensional activities of the brain. Indeed, in the context of presheaft theory, the cortical activity in 3D depends on the cortical activity in 4D. When a change in 3D occurs (either in source or target maps), we need to look for the foregoing changes in source and target maps in higher dimensions (Figure 5B). To provide an example, when a change takes place in the $3 \mathrm{D}$ source $\mathrm{s}_{3}$, we have to look for changes with matching description in $4 \mathrm{D}$, because:

$$
\mathrm{S}_{\mathrm{n}} \circ \mathrm{S}_{\mathrm{n}+1}=\mathrm{S}_{\mathrm{n}} \circ \mathrm{t}_{\mathrm{n}+1}
$$

Therefore, a change in $s_{3}$ is correlated with previous changes with the same features, either in $s_{4}$, or in $t_{4}$.

We showed that the theory of globular sets and presheaves might provide a well-grounded mathematical counterpart to nervous phenomena and suggested testable experimental previsions. Also, the approach in terms of globular sets paves the way to the theory of infinity categories, i.e., a mathematics founded on equivalence, rather than equality (Lurie 2003; Lurie 2008). In terms of brain activity, the concept of equality suggests that two neural waves are exactly the same, while the concept of equivalence suggests that two waves stand in relation to each other in many different ways. While equality is a strict relationship between two waves, equivalence comes in different forms correlated with 
homotopy paths between waves on the brain surface. This explains why apparently matching cortical oscillations give rise to highly different inter-individual responses. The last, but not the least, globular sets display affinities with conditional logic, i.e., a type of modal logic where several peculiar accessibility relations hold (Priest 2008). In particular, in touch with topological claims too (Ahmad and Peters, 2018; Peters 2019), analogies occur with the conditional $\operatorname{logic} \mathrm{C}$, in which validity is defined as truth preservation over all worlds of all interpretations. Stalnaker (1968) stated that the worlds accessible to the world w through $\mathrm{R}_{\mathrm{A}}$ - that is, the worlds essentially the same as w, except that $\mathrm{A}$ is true there - should be thought of as the worlds most similar to $\mathrm{w}$ at which $\mathrm{A}$ is true, with similarity coming by degrees. This suggests that nervous presheaves might be investigated in terms of conditional logic $\mathrm{C}$, and could explain why different perceptions come together in a single, whole percept. 
A

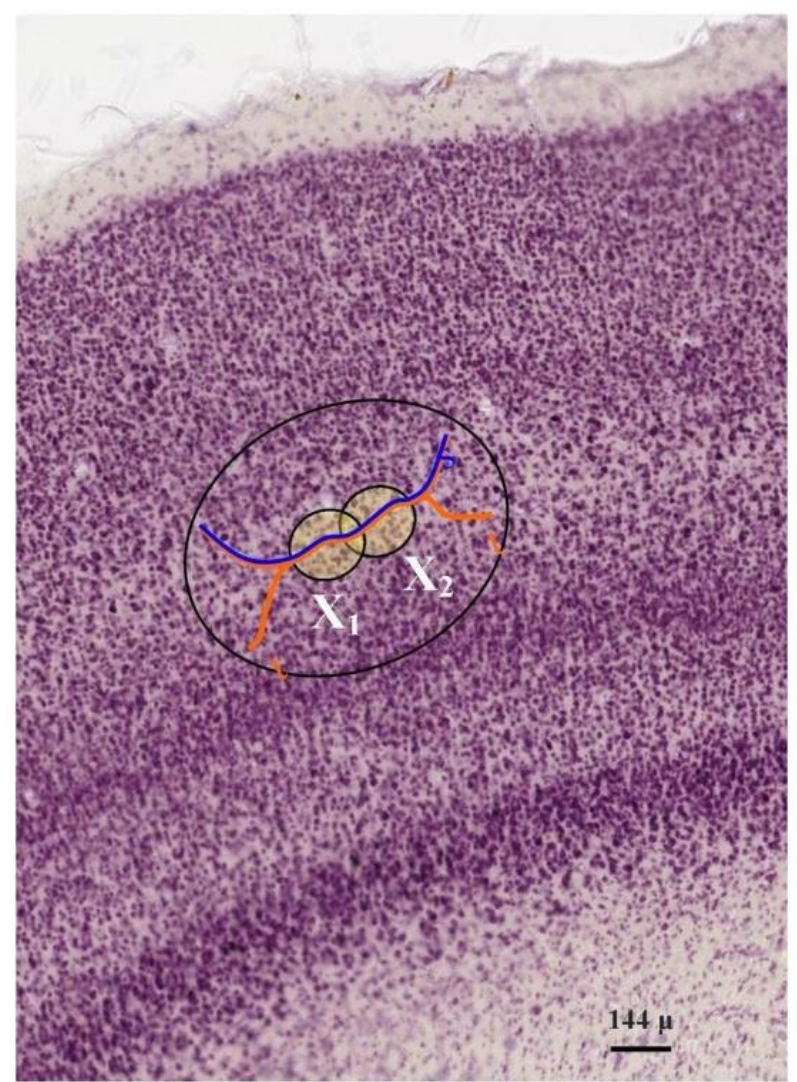

B
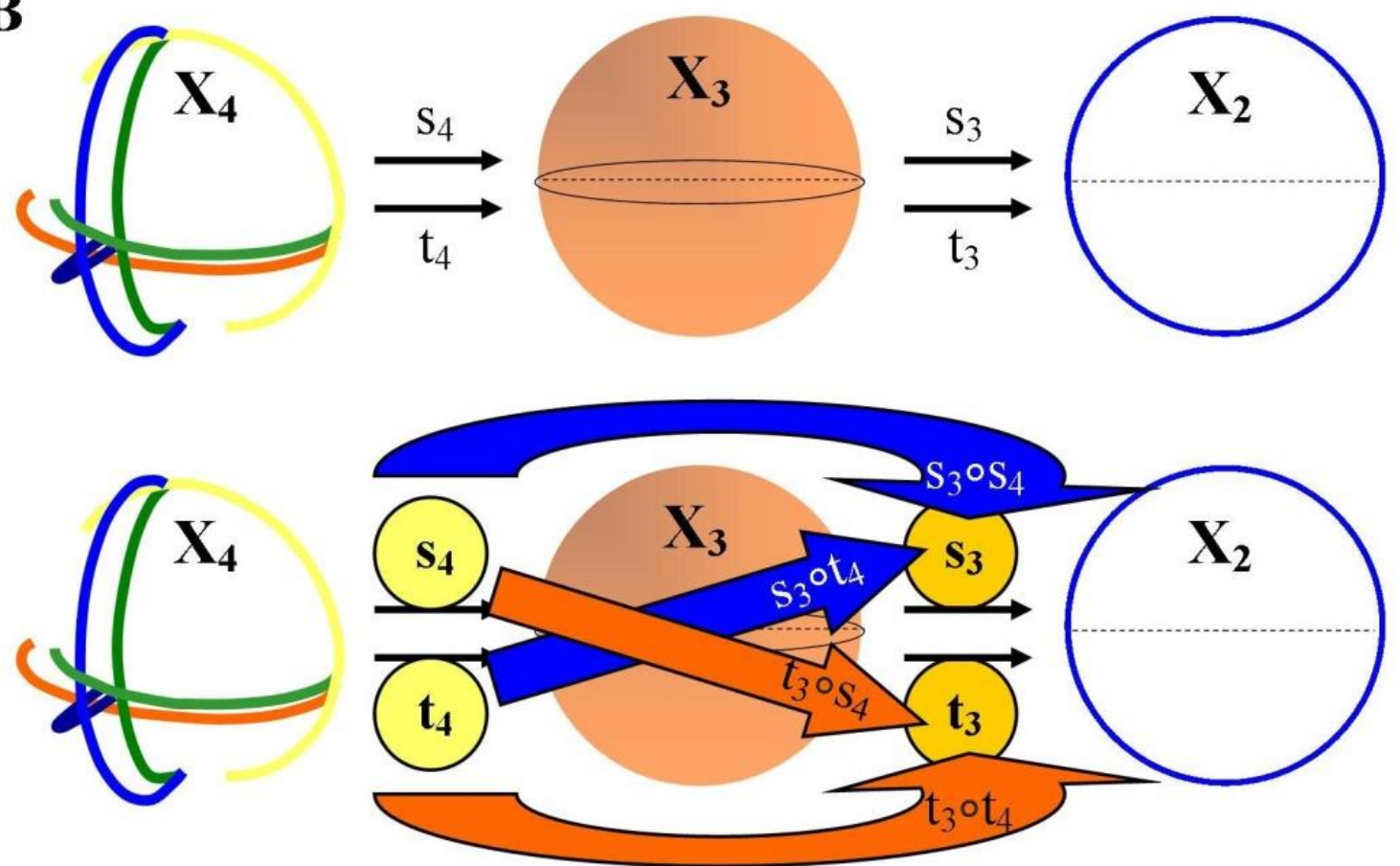

Figure 5. Application of presheaves in different nervous contexts. Figure 5A: Area 17 of Macaca mulatta potentially endowing globular sets in the granular layer IV. Modified from: http://brainmaps.org/ajaxviewer.php?datid $=2 \&$ sname $=0875$. Figure 5B. Globular sets encompassing manifolds of different spatial dimensions. Note how functions in higher dimensions precede functions in lower dimensions. The Figure shows how interdimensional projections map from an higher dimensional to a lower dimensional manifold. 


\section{REFERENCES}

1) Ahmad MZ, Peters JF. 2018. Descriptive unions. A fibre bundle characterization of the union of descriptively near sets, arXiv 1811, no. 11129, 1-19.

2) Axelrod V, Rozier C, Malkinson TS, Lehongre K, Adam C, et al. 2019. Face-selective neurons in the vicinity of the human fusiform face area. Neurology, 22;92(4):197-198. doi: 10.1212/WNL.0000000000006806.

3) Basser PJ, Roth BJ. 1991. Stimulation of a myelinated nerve axon by electromagnetic induction. Med Biol Eng Comput, 29(3):261-8.

4) Bastos AM, Vezoli J, Bosman CA, Schoffelen JM, Oostenveld R, et al. 2015. Visual areas exert feedforward and feedback influences through distinct frequency channels. Neuron. 2015 Jan 21;85(2):390-401. doi: 10.1016/j.neuron.2014.12.018.

5) Beleza P, Rémi J, Feddersen B, Peraud A, Noachtar S. 2010. Epidural and foramen-ovale electrodes in the diagnostic evaluation of patients considered for epilepsy surgery. Epileptic Disord. 2010 Mar;12(1):48-53. doi: 10.1684/epd.2010.0297.

6) Benali A, Trippe J, Weiler E, Mix A, Petrasch-Parwez E, et al. 2011. Theta-burst transcranial magnetic stimulation alters cortical inhibition. J Neurosci. 2011 Jan 26;31(4):1193-203. doi: 10.1523/JNEUROSCI.1379-10.2011.

7) Bergman C. 2011. Universal Algebra: Fundamentals and Selected Topics. CRC Press. ISBN 978-1-43985129-6.

8) Bredon GE. 1997. Sheaf Theory. Springer-Verlag New York. ISBN: 978-0-387-94905-5.

9) Chen R, Spencer DC, Weston J, Nolan SJ. 2016. Transcranial magnetic stimulation for the treatment of epilepsy. Cochrane Database Syst Rev, (8):CD011025. doi: 10.1002/14651858.CD011025.pub2.

10) Chung SW, Hill AT, Rogasch NC, Hoy KE, Fitzgerald PB. 2016. Use of theta-burst stimulation in changing excitability of motor cortex: A systematic review and meta-analysis. Neurosci Biobehav Rev.:43-64. doi: 10.1016/j.neubiorev.2016.01.008..

11) Chung SW, Sullivan C, Rogasch NC, Hoy KE, Bailey NW, et al. 2019. The effects of individualised intermittent theta burst stimulation in the prefrontal cortex: A TMS-EEG study. Hum Brain Mapp.;40(2):608627. doi: 10.1002/hbm.24398.

12) DeMaegd ML, Stein W. 2018. Long-Distance Modulation of Sensory Encoding via Axonal Neuromodulation. In: Sensory Nervous System. Edited by Thomas Heinbockel. IntechOpen DOI: 10.5772/intechopen.74647.

13) Dimca A. 2004. Sheaves in Topology. Springer-Verlag Berlin Heidelberg. ISBN: 978-3-642-18868-8.

14) Fields CA. 2013. The Principle of Persistence, Leibniz's Law, and the Computational Task of Object ReIdentification. Human Development, 56:147-166. https://doi.org/10.1159/000350206

15) Gersner R, Oberman L, Sanchez MJ, Chiriboga N, Kaye HL, et al. 2016. H-coil repetitive transcranial magnetic stimulation for treatment of temporal lobe epilepsy: A case report. Epilepsy Behav Case Rep. 2016; 5: 52-56. doi: 10.1016/j.ebcr.2016.03.001.

16) Guerra A, Suppa A, Bologna M, D'Onofrio V, Bianchini E, et al. 2018. Boosting the LTP-like plasticity effect of intermittent theta-burst stimulation using gamma transcranial alternating current stimulation. Brain Stimul.;11(4):734-742. doi: 10.1016/j.brs.2018.03.015.

17) Jan MM, Alsallum MS, Sadler MR. 2017. Transcranial Magnetic Stimulation and Epilepsy. International Journal of Medical Science and Clinical Inventions 4(10): 3256-3260. DOI:10.18535/ijmsci/v4i10.07 ICV 2015: 52.82.

18) Kashiwara M, Schapira P. 1994. Sheaves on manifolds, Grundlehren der Mathematischen Wissenschaften, 292, Berlin, New York: Springer-Verlag, ISBN 978-3-540-51861-7.

19) Kohli S, Casson A. 2019. Machine learning validation of EEG+tACS artefact removal. Journal of Neural Engineering, in press. https://doi.org/10.1088/1741-2552/ab58a3.

20) Komarov M, Malerba P, Golden R, Nunez P, Halgren E, Bazhenov M. 2019. Selective recruitment of cortical neurons by electrical stimulation. PLoS Comput Biol.;15(8):e1007277. doi: 10.1371/journal.pcbi.1007277.

21) Lurie J. 2003. On Infinity Topoi. arXiv:math/0306109.

22) Lurie J. 2008. Higher Topos Theory. arXiv:math/0608040.

23) McDermott B, Porter E, Hughes D, McGinley B1, Lang M, et al. 2018. Gamma Band Neural Stimulation in Humans and the Promise of a New Modality to Prevent and Treat Alzheimer's Disease. J Alzheimers Dis.;65(2):363-392. doi: 10.3233/JAD-180391.

24) Mejias JG, Murray JD, Kenned H, Wang X-J. 2016. Feedforward and feedback frequency-dependent interactions in a large-scale laminar network of the primate cortex. Science Advances 16 Nov 2016:, Vol. 2, no. 11, e1601335. DOI: 10.1126/sciadv.1601335. 
25) Michalareas g, Vezoli J, van Pelt S, Schoffelen J-M, Kennedy H, Fries P. 2016. Alpha-beta and gamma rhythms subserve feedback and feedforward influences among human visual cortical areas. Neuron. 2016 Jan 20; 89(2): 384-397. doi: 10.1016/j.neuron.2015.12.018.

26) Miller EK, Lundqvist M, Bastos AM. 2018. Working Memory 2.0. Neuron. 2018 Oct 24;100(2):463-475. doi: 10.1016/j.neuron.2018.09.023.

27) Northoff G, Tsuchiya N, Saigo H. 2019. Mathematics and the brain -a category theoretic approach to go beyond the neural correlates of consciousness. bioRxiv. doi: https://doi.org/10.1101/674242.

28) Papo D, Buldú JM. 2019. Brain synchronizability, a false friend. NeuroImage, 196: Pages 195-199. https://doi.org/10.1016/j.neuroimage.2019.04.029.

29) Peters JF. 2019. Vortex Nerves and their Proximities. Nerve Betti Numbers and Descriptive Proximity. arXiv:1910.08467.

30) Peters JF, Ramanna S, Tozzi A, İnan E. 2017. Bold-Independent Computational Entropy Assesses Functional Donut-Like Structures in Brain fMRI Images. Front Hum Neurosci. 2017 Feb 1;11:38. doi: 10.3389/fnhum.2017.00038.

31) Priest G. 2008. An Introduction to Non-Classical Logic, Second Edition: From If to Is. Cambridge University Press. ISBN-13: 978-0521670265.

32) Rattay F. 1986. IEEE Trans Biomed Eng, 33(10):974-7.

33) Rattay F, Paredes LP, Leao RN. 2012. Strength-duration relationship for intra- versus extracellular stimulation with microelectrodes. Neuroscience. 2012 Jul 12;214:1-13. doi: 10.1016/j.neuroscience.2012.04.004.

34) Santarnecchi E, Sprugnoli G, Bricolo E, Costantini G, Liew S, et al. 2019. Gamma tACS over the temporal lobe increases the occurrence of Eureka! moments. Sci Rep.;9(1):5778. doi: 10.1038/s41598-019-42192-z.

35) Sikkens T, Bosman CA, Olcese U. 2019. The Role of Top-Down Modulation in Shaping Sensory Processing Across Brain States: Implications for Consciousness. Front. Syst. Neurosci. https://doi.org/10.3389/fnsys.2019.00031.

36) Simpson C. 2011. Homotopy Theory of Higher Categories - From Segal Categories to n-Categories and Beyond. Cambridge University Press. ISBN:9780511978111.

37) Stalnaker R. 1968. A Theory of Conditionals. In Studies in Logical Theory, American Philosophical Quarterly Monograph Series, no. 2. Oxford: Basil Blackwell.

38) Street R. 2000. The petit topos of globular sets. Journal of Pure and Applied Algebra 154, (1-3): 299-315. https://doi.org/10.1016/S0022-4049(99)00183-8.

39) Tangermann M, Navin Lal T, Hinterberger T, Bogdan M, Hill NJ, et al. 2005. Robust EEG Channel Selection across Subjects for Brain-Computer Interfaces. Computer SciencePublished in EURASIP J. Adv. Sig. Proc. DOI:10.1155/ASP.2005.3103.

40) Tozzi A, Peters JF. 2016. Towards a Fourth Spatial Dimension of Brain Activity. Cognitive Neurodynamics 10 (3): 189-199. doi:10.1007/s11571-016-9379-z.

41) Tozzi A, Peters JF, Fingelkurts AA, Fingelkurts AA, Marijuán PC. 2017a. Topodynamics of metastable brains. Physics of Life Reviews, 21, 1-20. http://dx.doi.org/10.1016/j.plrev.2017.03.001.

42) Tozzi A, Peters JF, Ori O. 2017b. Cracking the barcode of fullerene-like cortical microcolumns. Neurosci Letters, 644, 100-106. http://dx.doi.org/10.1016/j.neulet.2017.02.064.

43) Tozzi A. 2019. The multidimensional brain. Physics of Life Reviews. In press. doi: https://doi.org/10.1016/j.plrev.2018.12.004.

44) Wolski M. 2013. Toward foundations of near sets: (pre-)sheaf theoretic approach. Math. Comput. Sci. 7(1), 125-136, MR3043923.

45) Zheng C, Colgin LL. 2015. Beta and gamma rhythms go with the flow. Neuron. 2015 Jan 21; 85(2): 236237. doi: 10.1016/j.neuron.2014.12.067. 